\title{
CONTINUOUS COHOMOLOGY OF GROUPS AND CLASSIFYING SPACES
}

\author{
BY JAMES D. STASHEFF ${ }^{1}$
}

Topological groups exhibit one of the richest structures in mathematics, both because of the variety of significant examples and because of the interplay between topology and algebra. The examples range through geometry and into the study of differential equations. Indeed when Sophus Lie began to look at continuous groups around 1870 [35], he was particularly interested in those respecting a geometric structure and those respecting the solutions of a differential equation. By considering the solution sets of the equation as topological objects, it is possible to combine these two aspects; this is precisely a point of view now current in the theory of foliations. Following Lie, one finds interest not only in Lie groups but in Lie groupoids, for example, of local diffeomorphisms of a manifold.

To an algebraic topologist, there is a special challenge in the interplay between the algebra and topology of a topological group or groupoid. He can ignore the algebra and consider the cohomology of the underlying space or he can ignore the topology and consider the cohomology of the abstract group, but clearly neither is a satisfactory approach to these objects. To combine both the topology and the algebra, he has a variety of possibilities.

For any group $G$, let $G^{\delta}$ denote the corresponding group with the discrete topology. In particular, any abstract group can be thus topologized.

Looking at the cohomology of abstract groups as defined in terms of multivariable functions on the group, he can restrict attention to the continuous functions when dealing with a topological group $G$. This results in the continuous (group theoretic) cohomology $H_{c}(G)$.

Alternatively, since the abstract group cohomology $H(G)=H_{c}\left(G^{\delta}\right)$ is isomorphic with that of an associated space $B G^{\delta}$, he can carry the topology into the construction of a corresponding classifying space $B G$ and then consider $H(B G)$.

Finally for Lie groups, he can also consider the associated Lie algebra $g$ and the Lie algebraic cohomology $H(\mathfrak{g})$.

An expanded version of an invited address presented to the 79th Summer Meeting of the American Mathematical Society in Kalamazoo, Michigan on August 21, 1975; received by the editors April 25, 1977.

AMS (MOS) subject classifications (1970). Primary 55F35, 55F40, 55B35, 55H99; Secondary 17B65, 18H25, 18H10, 57F15.

Key words and phrases. Continuous cohomology, topological group, Lie group, Lie algebra cohomology, Bott spectral sequence, $\Gamma_{n}$, foliations, Gel'fand-Fuks cohomology, classifying space.

'Supported in part by NSF Grant 23976 and the generous hospitality of the Institute for Advanced Study. 
Today I would like to survey what is known about the relations between these various cohomologies. Many of the results date from the early 1950 's, but some of the relations involving $B G$ are quite recent and they are the primary import of this account. Beyond that, the applications to Lie groupoids, foliations and infinite dimensional Lie algebras are the focus of much current activity; I have tried to make this account as up to date as possible. (An alternate approach to much of this material has appeared in lecture notes of Haefliger [67].)

The various parts of the paper are organized in terms of the appropriate domain category for the cohomology functor in question.

81. The abstract (discrete) case. A review of abstract group cohomology.

82. Continuous cohomology of groups and extensions. Formal definition and an interpretation of $H_{c}^{2}(G: A)$ in terms of extensions of topological groups.

83. Long extensions and continuous homological algebra. An interpretation of $H_{c}(G ; A)$ from the point of view of homological algebra.

84. Borel cohomology. A brief account of the measure theoretic generalization appropriate to locally compact groups. Here it is now possible to handle the topology axiomatically.

85. Lie algebra cohomology. A review of the results of van Est relating $H_{c}(G)$ to $H(g ; k)$ and those of Chevalley-Eilenberg with regard to $H\left(G_{U} / K\right)$.

86. Classifying spaces. The construction and the relation to $H_{c}(G)$. The Bott spectral sequence between $H_{c}\left(G ; S^{*}(\overline{\mathfrak{g}})\right)$ and $H(B G)$ with particular attention to $G=G L(n, R)$.

87. Continuous cohomology of topological groupoids. The generalization of $\$ \$ 2$ and 6 to Lie groupoids with particular attention to $\Gamma_{n}$, the groupoid of germs of local diffeomorphisms of $R^{n}$. The Bott vanishing theorem and the algebra $H\left(W O_{n}\right)$ of characteristic classes for foliations.

88. Continuous cohomology of vector fields. A brief account of the continuous cohomology of infinite dimensional Lie algebras, e.g. $\mathscr{X}(M)$, the algebra of vector fields on a manifold, and the relation to $B \Gamma_{n}$.

89. Coda. A description of the relation between $H_{c}(G), H(B G)$ and the cohomology of the discrete (abstract) group $G^{\delta}$, including the two topologies approach of Bott and Haefliger. In particular, $H_{c}(G)$ is literally intermediate between $H\left(G^{\delta}\right)$ and $H(B G)$ : we have

$$
H\left(G^{\delta}\right) \approx H\left(B G^{\delta}\right) \leftarrow H_{c}(G) \leftarrow H(B G) .
$$

Also the relation to $H_{\text {Borel }}(G ; R / Z)$ via differential characters.

1. The abstract (discrete) case [30]. Consider an abstract group $G$ acting on a space $E$ so that the quotient map $E \rightarrow E / G$ is a covering space. If $E$ has trivial homology and is simply connected, the homology of $E / G$ depends only on the group $G$, which appears as the fundamental group of $E / G$. Hopf showed the homology of $E / G$ depends only on the group and expressed the dependence algebraically [68, cf., p. 137]. Eilenberg and Mac Lane did so independently [40] by constructing a particular simplicial space $E / G$ with $n$-simplices labelled by $n$-tuples of elements of $G$. The labelling pattern is 
indicated in the following picture: There is only one 0-simplex * identified with all the vertices $0,1, \ldots$.

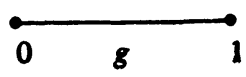

A 1-simplex $g$

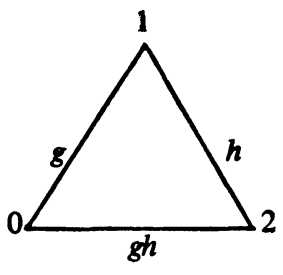

A 2-simplex $(g, h)$

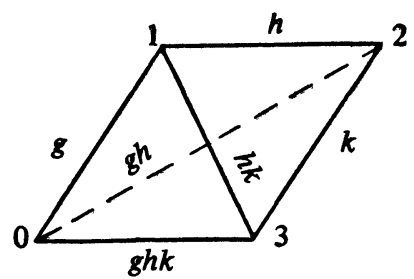

A 3-simplex $(g, h, k)$

Continuing this pattern produces an infinite dimensional complex; for the group $G=Z / 2$, the result is $R P(\infty)$, up to homotopy type.

Alternatively, this combinatorial structure yields a coboundary formula which can be expressed purely group theoretically:

The group of $p$ cochains $C^{P}(G ; A)$ with coefficients in a right $G$-module $A$ is the group of all functions

$$
f: \underbrace{G \times \cdots \times G}_{p} \rightarrow A
$$

with coboundary $\delta: C^{p}(G ; A) \rightarrow C^{p+1}(G ; A)$ defined by

$$
\begin{aligned}
(\delta f)\left(g_{1}, \ldots, g_{p+1}\right) & =f\left(g_{2}, \ldots, g_{p+1}\right) \\
& +\sum(-1)^{i} f\left(g_{1}, \ldots, g_{i} g_{i+1}, \ldots, g_{p+1}\right) \\
& +(-1)^{p+1} f\left(g_{1}, \ldots, g_{p}\right) \cdot g_{p+1} .
\end{aligned}
$$

It is straightforward to verify $\delta \delta=0$.

The cohomology groups were defined as $\operatorname{Ker} \delta / \operatorname{Im} \delta$ and $H^{0}, H^{1}, H^{2}, H^{3}$ could be related to algebraically significant concepts: invariant elements, crossed homomorphisms, factor sets for extensions and Teichmüller cocycles. An excellent summary of these developments appears in Mac Lane's Retiring Presidential Address [39, 83].

2. Continuous cohomology of groups and extensions. Now for topological groups, one could mimic these procedures, but there turns out to be a choice of where to insert the topology. In the work of Heller [28], Hu [31] and van Est [62], it first went into the formal definition.

For a topological group, the continuous cohomology $H_{c}(G ; A)$ with coefficients in a continuous $G$-module $A$ is that of the cochain groups

$$
C_{c}^{p}(G ; A)=\left\{\text { continuous } f: G^{p} \rightarrow A\right\}
$$

with the above coboundary. Here $A$ itself has a topology (e.g. $A=R$, the real numbers) and the action $A \times G \rightarrow A$ is assumed continuous, (e.g. $A=$ $\left.C^{\infty}(G)\right)$.

$H^{0}$ and $H^{1}$ behave entirely as expected, but for $H^{2}$ life becomes more interesting. Abstract group extensions are always split as sets, in contrast to the topological case. Hu in 1951 [31] (and independently Heller) showed that $H_{c}^{2}(G ; A)$ classified topologically split group extensions, i.e. short exact sequences 


$$
1 \rightarrow A \rightarrow E \rightarrow G \rightarrow 1
$$

such that, as a space, $E$ is $A \times G$. Thus if we write elements of $E$ as pairs $(a, g)$, a continuous two-cocycle $f: G \times G \rightarrow A$ determines a continuous multiplication by

$$
(a, g)\left(a^{\prime}, g^{\prime}\right)=\left(\left(a \cdot g^{\prime}\right) a^{\prime} f\left(g, g^{\prime}\right), g g^{\prime}\right) .
$$

More generally, one can consider locally split extensions; that is, as spaces, $E \rightarrow G$ is a principal $A$-bundle [28]. Indeed for finite dimensional locally compact separable, metric groups, any extension with $A$ closed in $E$ is locally split [47].

Dennis Johnson and Graeme Segal [53] showed independently that in general $\delta \operatorname{xt}(G ; A)$, the set of equivalence classes of extensions

$$
1 \rightarrow A \rightarrow E \rightarrow G \rightarrow 1
$$

which are principal $A$-bundles, is described by an exact sequence

$$
H_{c}^{2}(G ; A) \rightarrow \mathcal{E x t}(G ; A) \rightarrow \check{H}^{1}(G ; \mathbf{A}),
$$

the last group being the Čech cohomology of the space $G$ with coefficients in the sheaf of continuous functions of $G$ with values in $A$. Segal mentioned in a letter (1970) that the image in $\check{H}^{1}$ is primitive and the sequence extends:

$$
H_{c}^{2}(G ; A) \rightarrow \mathcal{E x t}(G ; A) \rightarrow \operatorname{Prim} \check{H}^{1}(G ; \mathrm{A}) \rightarrow H_{c}^{3}(G ; A) .
$$

A class $u$ is primitive if, under the multiplication $m: G \times G \rightarrow G$, the class $u$ pulls back to $u \times 1+1 \times u$ in $H^{1}(G \times G$; A). Such a class determines a bundle $E$ which admits a multiplication; the image in $H_{c}^{3}$ is the obstruction to associativity. (A curiously similar result is known for extensions of $H$-spaces [57].)

Using different techniques, both Johnson and Segal obtain the above exact sequence as a corollary of a spectral sequence. For Johnson, the starting point is a bicomplex combining the Čech and Eilenberg-Mac Lane coboundaries. This approach has been rediscovered by Jean-Louis Cathelineau [12].

As one might hope, given a topological group $G$ and a closed normal subgroup $H$, there is usually a continuous version of the Hochschild-Serre spectral sequence from $H_{c}\left(G / H ; H_{c}(H ; A)\right)$ to $H_{c}(G ; A)$ [61], [29], [13], [4], although even for Lie groups there are real subtleties if $A$ is infinite dimensional.

3. Long extensions and continuous homological algebra. In the discrete case, although $H^{2}(G ; A)$ is related nicely to extensions $1 \rightarrow A \rightarrow E \rightarrow G \rightarrow 1$, the higher cohomology groups $H^{n}(G ; A)$ are more readily interpreted in terms of long exact sequences

$$
0 \rightarrow A \rightarrow E_{1} \rightarrow \cdots \rightarrow E_{n} \rightarrow Z \rightarrow 0
$$

of $G$-modules. The same holds true in the topological case, with some restriction on the kinds of exact sequences considered.

Let $M_{g}$ denote the category of topological $G$-modules. Let $\mathfrak{N}$ denote a distinguished class of short exact sequences to be called proper (rel $\Re)$. Define a long exact sequence

$$
0 \rightarrow E_{0} \stackrel{f_{0}}{\rightarrow} E_{1} \rightarrow \cdots \rightarrow E_{n} \stackrel{f_{n}}{\rightarrow} E_{n+1} \rightarrow 0
$$


to be proper (rel $\Re$ ) if it can be decomposed as a splicing of proper short exact sequences. Equivalence classes are defined in terms of the generating relation: two proper sequences $\left\{E_{i}\right\}$ and $\left\{D_{i}\right\}$ as in (*) are equivalent if $D_{0}=D_{0}$ and $E_{n+1}=D_{n+1}$ and there is a commutative diagram

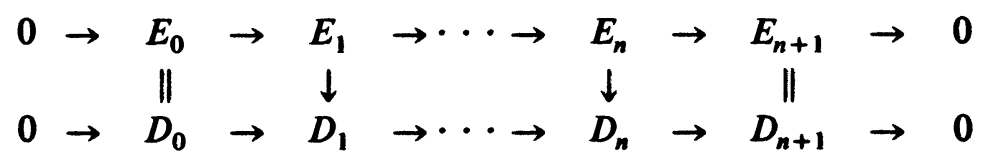

DefinItion. Ext ${ }_{\mathscr{N}}^{n}(C ; A)$ will denote the set of equivalence classes of proper (rel $\Re$ ) sequences (*) with $A=E_{0}$ and $C=E_{n+1}$. (In fact, $\operatorname{Ext}_{\Re /}^{n}(C ; A)$ can be given the structure of an abelian group in the usual way [38] by considering the Baer sum of extensions.)

As in our discussion of $H_{c}^{2}$, the appropriate class $\pi$ is $\delta$, the class of topologically split short exact sequences, i.e. $0 \rightarrow A \rightarrow B \stackrel{p}{\rightarrow} C \rightarrow 0$ is proper if and only if $p$ has a continuous section $\sigma: C \rightarrow B$. The situation is quite categorical; the section permits the classical (discrete) proofs to be followed almost verbatim.

The main result is:

For any topological group $G$ and topological $G$-module $A$, we have a natural isomorphism

$$
H_{c}^{n}(G ; A) \approx \operatorname{Ext}_{\delta}^{n}(Z ; A) .
$$

This is explicit in Heller [28] for $n=2$ and implied in general in both Heller and Wigner [66].

By restricting the groups involved, the class $\Re$ can be expanded. For example consider the class $\theta$ of short exact sequences $0 \rightarrow A \rightarrow B \stackrel{p}{\rightarrow} C \rightarrow 0$ in which $p$ is open.

THEOREM [66]. If $G$ is locally compact, $\sigma$-compact then

$$
H_{c}^{n}(G ; A) \approx \operatorname{Ext}_{\mathfrak{O}}^{n}(Z ; A)
$$

provided (1) $G$ is also 0-dimensional or (2) $G$ is finite dimensional and $A$ is, as a group, a vector space.

A closely related description of the cohomology of abstract groups is in terms of "resolutions". Our definition of $H_{c}(G ; A)$ uses essentially a specific resolution of $Z$ by free $G$-modules. Alternatively the same answer can be obtained in many cases by resolving the coefficients $A$. This approach was carried out by Hochschild and Mostow [29], following the discrete model quite neatly. Again one works with the class $\mathcal{S}$ of topologically split short exact sequences of $G$-modules and uses the machinery of relative homological algebra, e.g. a module is $\delta$-injective if it is injective with respect to exact sequences proper rel $\delta$ and a resolution is a proper rel $\delta$ exact sequence. The expected result is:

THEOREM [29]. If $G$ is a topological group and $A$ is a topological G-module, then $H_{c}(G ; A)$ is isomorphic to the cohomology of the invariant subcomplex $0 \rightarrow A^{G} \rightarrow X_{0}^{G} \rightarrow X_{1}^{G} \rightarrow \cdots$ of any $\mathcal{S}$-injective resolution of $A$. (Equivalently, $H_{c}(G ; A)$ is the relative derived functor of the submodule $A^{G}$.) 
This approach remembers the topology enough to give $H_{c}(G ; A)$ the structure of a topological $G$-module, at least if $G$ is locally compact. A particularly neat summary is given by Casselman and Wigner [13].

4. Borel cohomology. The above discussion emphasizes the relevance of $\boldsymbol{H}_{c}$ to topologically split extensions. For locally compact groups an alternate construction called Borel cohomology $H_{\text {Borel }}(G ; A)$ is more widely relevant.

As initiated by Mackey and fully defined by C. C. Moore [43], the definition is that of $H_{c}$ in $\$ 2$ except that the functions

$$
f: G \stackrel{p}{\rightarrow} A
$$

are only required to be Borel (measurable) with respect to the $\sigma$-field of open sets, that is, the collection of sets generated by countable intersections of open sets.

THEOREM (MACKEY [37]). If $G$ and $A$ are locally compact, then

$$
H_{\text {Borel }}^{2}(G ; A) \approx \operatorname{Ext}(G ; A),
$$

the group of equivalence classes of all topological group extensions.

The point is that topological group extensions of locally compact groups are Borel split. [L. G. Brown [10] has extended these results to polonais groups.]

Wigner looks at extensions proper rel $\theta$, the class of short exact sequences in which $p$ is open.

THEOREM [66]. If $G$ is Hausdorff and all topological $G$-modules are complete metrizable, then

$$
H_{\text {Borel }}^{2}(G ; A) \approx \operatorname{Ext}_{\Theta}(Z, A) .
$$

For Borel cohomology, C. C. Moore [43, III] has developed a short set of axioms wich characterize the cohomology functor for a reasonable category of groups and modules. A topological group is polonais if and only if it admits a separable complete metric. Associated to any topological $G$-module $A$, we have $I(A)$, the $G$-module of equivalence classes of Borel functions $G \rightarrow A$ (two are equivalent if they agree almost everywhere), which will play the role of the regular representation.

Definition [43, III, p. 16]. A cohomological functor on a category $\Re(G)$ of $G$-modules is a sequence $H^{n}(G ; A)$ of covariant functors from $\mathfrak{T}(G)$ to abelian groups such that

(1) for all $0 \rightarrow A^{\prime} \rightarrow A \rightarrow A^{\prime \prime} \rightarrow 0$ in $\Re(G)$ there is a natural transformation $\partial: H^{n}\left(G ; A^{\prime \prime}\right) \rightarrow H^{n+1}\left(G ; A^{\prime}\right)$ such that the usual long exact cohomology sequence holds

(2) $H^{0}(G ; A)=A^{G}$, the $G$-fixed points of $A$,

(3) $H^{n}(G ; I(A))=0$ for all $r \geqslant 1$ and all $A$.

For separable locally compact groups $G$, Moore proves $H_{\text {Borel }}$ is the unique cohomology functor on $P(G)$, the category of polonais $G$-modules. The vanishing condition of axiom (3) permits systematic application of many of the standard techniques of homological algebra. 
In relation to classifying spaces, we will see later that both $H_{\text {Borel }}$ and $H_{c}$ are relevant, depending on the coefficient module.

5. Cohomology of Lie algebras. As for computing $H_{c}(G ; A)$, the greatest success is for Lie groups. Just as the singular cohomology of a space is often computable because it agrees with a finite version (simplicial or cellular), so the continuous cohomology of a Lie group is often computable because it agrees with the cohomology of a corresponding (finite dimensional) Lie algebra pair.

As usual, there is a variety of definitions of Lie algebra cohomology. We choose to define it in terms of a specific complex, emphasizing the similarity with our definition of continuous cohomology.

Let $g$ be a Lie algebra over a field $k$ and $\bar{g}=\operatorname{Hom}(G, k)$ its vector space dual. Let $\Lambda^{*} \bar{g}=\bigoplus \Lambda^{q} \bar{g}$ be the exterior algebra generated by $\bar{g}$. Equivalently $\Lambda^{q} \bar{g} \approx \operatorname{Alt}^{q}(g ; k)$, the space of alternating multilinear forms of $q$ variables, for finite dimensional $g$.

Now let $A$ be a module over the Lie algebra $g$; that is, $A$ is a vector space over $k$ with a representation $Q: g \rightarrow$ End $(A)$ such that $Q[X, Y]=$ $Q(X) Q(Y)-Q(Y) Q(X)$. In particular if $A$ is a $G$-module, $\pi: G \rightarrow G L(A)$, it is also a $g$-module by $d \pi: g \rightarrow g l(A)$.

The cochain group $C^{q}(g ; A)$ is $\operatorname{Alt}^{q}(g ; A)$ which for finite dimensional $g$ can be identified with $\Lambda^{q}(\bar{g}) \otimes A$. Define

$$
\delta: C^{q}(g ; A) \rightarrow C^{q+1}(g ; A)
$$

by

$$
\begin{aligned}
(\delta w)\left(X_{0}, \ldots, X_{q}\right)= & \sum_{i<j}(-1)^{i+j} w\left(\left[X_{i}, X_{j}\right], \ldots, \hat{X}_{i}, \ldots, \hat{X}_{j}, \ldots\right) \\
& +\sum(-1)^{i} X_{i} w\left(X_{1}, \ldots, \hat{X}_{i}, \ldots, X_{q}\right) .
\end{aligned}
$$

After verifying that $\delta \delta=0$, let $H(g ; A)$ be the resulting cohomology:

$$
H^{q}(g ; A)=\frac{\operatorname{Ker} \delta \mid C^{q}(g ; A)}{\operatorname{Im} \delta C^{q-1}(g ; A)} .
$$

For a pair of Lie algebras $h \subset g$, define the relative cochain groups $C^{q}(g, h ; A)$ to consist of those cochains $w \in C^{q}(g ; A)$ such that

(1) $w\left(X_{1}, \ldots, X_{q}\right)=0$ if some $X_{i} \in h$,

(2) $w\left(X_{1}, \ldots,\left[X_{i}, Y\right], \ldots, X_{q}\right)=Y \cdot w\left(X_{1}, \ldots, X_{q}\right)$

if $Y \in h$.

The above coboundary $\delta$ respects these conditions and the relative cohomology is denoted $H^{q}(g, h ; A)$.

Among other important relations, van Est (1953) [62] establishes:

If $G$ is a connected Lie group with $K \subset G$ a maximal compact subgroup, and if $k \subset g$ are the corresponding Lie algebras, then for $A$, a vector space over $R$, we have

$$
H_{c}(G ; A) \approx H(g, k ; A) .
$$

Actually van Est used smooth cochains. Part of the point of the work of Mostow [48] and Hochschild and Mostow [29] was to generalize this result to 
continuous cochains and more general coefficients, e.g. locally convex integrable differential $\boldsymbol{A}$. On the other hand, Chevalley and Eilenberg [15] formalized results of $\mathrm{E}$. Cartan relating Lie algebra cohomology to the topological spaces: If $K \subset G$ are connected Lie groups with $G$ compact and Lie algebras $k \subset g$, then

$$
H(g, k ; A) \approx H(G / K ; A) .
$$

If $G$ and $K$ are not connected, one has to introduce the slight modification denoted $H(g, K ; A)$ defined by replacing (2) above by

$\left(2^{\prime}\right) w$ is invariant under the action of $K$.

If $G$ is not compact we need to assume there is at least some "compact form" $G_{u}$, that is a compact connected Lie group having the same Lie algebra, at least after complexification. The notation $G_{u}$ reflects the fact that $G_{u}$ can best be described in terms of unitary representations. The compact form need not be of the same homotopy type. For $G=G L(n, R)$, a compact form $G_{u}$ is $U(n)$.

With these modifications combined with the van Est result, we have for a finite dimensional Lie group $G$ :

$$
H_{c}(G ; R) \approx H\left(G_{u} / K ; R\right)
$$

where $K$ is maximal compact in $G$. For example

$$
H_{c}(G L(n, R) ; R) \approx \Lambda\left(h_{1}, h_{3}, \ldots, h_{\langle n\rangle}\right) \approx H(U(n) / O(n) ; R)
$$

where degree $h_{i}$ is $2 i-1$ and $\langle n\rangle$ is the largest odd integer $\leqslant n$. In contrast $H C(K)=H_{c}(O(n))=R$ since $K=O(n)$ is already compact.

Over a $p$-adic field instead of $R$, the results are startlingly different. Lazard [34] has shown that over $Q_{p}$, the $p$-adic rationals, for $G$ the rational points of a connected semisimple group

$$
H_{c}\left(G ; Q_{p}\right) \approx H^{*}\left(g ; Q_{p}\right) .
$$

The result has been extended further by Casselman and Wigner [13]

Outside of the case of Lie (including discrete) groups, I know of only one other class of groups for which we have computations of the algebra $H_{c}(G)$ : for profinite groups $G$, Serre [54] shows that with discrete coefficients $A$,

$$
H_{c}(G ; A) \approx \text { ind } \lim H\left(G / U ; A^{U}\right)
$$

when $U$ runs over all distinguished open subgroups of $G$. (Special cases of $H_{c}^{2}(G ; A)$ have been computed, e.g. [43] for compact groups as limits of Lie groups.) Perhaps the time is ripe for progress with this problem.

6. Classifying spaces. Now let us look again at how the cohomology of groups got started. The space $E / G$ was built from a set of simplices indexed by tuples from $G$ so, if $G$ has a topology, we can introduce it into the index set. That is, we define [42], [16], [41], [56].

$$
B G=\Perp \Delta^{n} \times G^{n} / \sim
$$

where the equivalence relation is embodied in the (co)boundary formula and the pictures of $\S 1$. To be precise, 


$$
\begin{aligned}
\left(t_{0}, \ldots, t_{p}, g_{1}, \ldots, g_{p}\right) \underset{t_{0}}{\sim} \underset{\sim}{\sim}\left(t_{1}, t_{2}, \ldots, g_{2}, \ldots, g_{p}\right) \\
\underset{t_{i}=0}{\sim}\left(t_{0}, \ldots, t_{i}, \ldots, g_{i} g_{i+1}, \ldots, g_{p}\right) \\
\underset{t_{n}=0}{\sim}\left(t_{0}, \ldots, t_{p-1}, g_{1}, \ldots, g_{p-1}\right) .
\end{aligned}
$$

It is worth noting that the equivalence relation is well defined because of the associativity.

If $G=S^{1}$, then $B G$ is $C P(\infty)$, quite an appropriate generalization of $G=Z / 2$ and $B G=R P(\infty)$.

Obviously $B G$ incorporates in its topology both the topology and algebra of $G$. One reflection of this is that $G$ has the homotopy type of the space of loops $\Omega B G$ on $B G[16]$. Thus $H(B G ; A)$ is an appropriate cohomology of the topological group $G$. Until further notice, we will use $A=R$ and drop it from the notation.

Notice that whereas $H\left(G^{\delta}\right)=H\left(B G^{\delta}\right)$ in the discrete case, we have, with real coefficients, $H_{c}\left(S^{1}\right)=0$ (since $S^{1}=S_{u}^{1}$ and is its own maximal compact subgroup) while $H\left(B S^{1}\right)=H(C P(\infty))=R[u]$ where $u$ has dimension 2.

How then are $H_{c}(G)$ and $H(B G)$ related? And how is either related to $H_{\text {top }}(G)$, the cohomology of the underlying space? The latter question can be answered by looking at how $B G$ is built up from pieces $\Delta^{n} \times G^{n}$ (as $C P(\infty)$ is built as a union of even dimensional cells: $\left.* \cup e^{2} \cup e^{4} \cup \cdots\right)$. The computations can be summarized for coefficients $R$ (or any field) more readily in terms of homology. The multiplication on $G$ induces the structure of an algebra on $H_{*}^{\text {top }}(G)$; the homology of that algebra can then be considered. There is a special sequence [56], [45] going from

$$
H_{*}^{\mathrm{alg}}\left(H_{*}^{\mathrm{top}}(G)\right) \stackrel{\text { def }}{=} \text { Tor } H_{*}^{\text {top }}(G) \text { to } H_{*}(B G) .
$$

For example, again with real coefficients, the homology algebra of any finite dimensional Lie group is an exterior algebra on odd dimensional generators $\Lambda\left(x_{1}, \ldots, x_{n}\right)$ and $H_{*}^{\text {alg }} H_{*}^{\text {top }}(G)=H_{*}(B G)$, dual to the cohomology algebra $R\left[y_{1}, \ldots, y_{n}\right]$ where

$$
\operatorname{dim} y_{i}=\operatorname{dim} x_{i}+1 \text {. }
$$

We return to cohomology to consider $H_{c}$ since there is no good continuous homology theory at present. For real coefficients, there is a nice map

$$
H^{*}(B G ; R) \rightarrow H_{c}^{*}(G ; R) \text {. }
$$

From the description of $B G$ as the quotient of $\Perp \Delta^{p} \times G^{p}$, there are certain obvious singular simplices of $B G$, i.e.

$$
\Delta\left(g_{1}, \ldots, g_{p}\right): \Delta^{p} \rightarrow \Delta^{p} \times\left(g_{1}, \ldots, g_{p}\right) \rightarrow \Delta^{p} \times G^{p} \rightarrow B G .
$$

Given a singular cochain $u: C_{*}(B G) \rightarrow A$, the restriction to $\Delta\left(g_{1}, \ldots, g_{p}\right)$ induces a function

$$
u \circ \Delta: G^{p} \rightarrow A
$$

and the topological coboundary corresponds precisely to the group theoretic one if we use $C^{*}(B G ; \mathbf{A})$ where $\mathbf{A}$ is the locally constant sheaf (local 
coefficient system) corresponding to the $\pi_{1}(B G)=G$-module structure on $A$. In general $u \circ \Delta$ will be continuous, so we restrict attention to the subcomplex $C_{c}^{*}(B G ; \mathbf{A})$ of cochains $u$ which are continuous in terms of the compact-open topology for $(B G)^{\Delta^{n}}$. We then have

$$
H(B G ; \mathrm{A}) \leftarrow H_{c}(B G ; A) \rightarrow H_{c}(G ; A)
$$

where the right-hand map is induced by $u \rightarrow u \circ \Delta$.

If $G$ has the homotopy type of a CW-complex and $A=R$ with the trivial action, then the left-hand map is an isomorphsm according to Mostow [49]. (An earlier result like this, recorded by $\mathrm{Hu}$ [30], works for compact Hausdorff spaces and Alexander-Spanier cohomology.)

For discrete coefficients, Borel cohomology works better.

THEOREM (WIGNER, [66]). For $A$ discrete and $G$ finite dimensional locally compact, $\sigma$-compact

$$
H(B G ; A) \approx H_{\text {Borel }}(G ; A) .
$$

Now for Lie groups and real coefficients, the identification of $H_{c}(G)$ with $H\left(G_{u} / K\right)$ allows us to relate $H_{c}(G)$ and $H(B G)$ via the spectral sequence of the fibration

$$
G_{u} / K \rightarrow B K \rightarrow B G_{u}
$$

where $K$ being maximal in $G$ implies $B K \simeq B G$. Essentially the same relationship can be obtained more intrinscially from the construction of $B G$, as was done by Bott:

For a Lie group $G$, there is a spectral sequence from

$$
H_{c}^{*}\left(G ; S^{*}(\bar{g})\right) \text { to } H^{*}(B G)
$$

where $S$ denotes the symmetric algebra and $\bar{g}$ the dual of the Lie algebra. The idea is simple enough. The de Rham version [9], [17], [65] of an old singular result [56] asserts $H(B G)$ is given by the double complex

$$
\Omega^{*}\left(G^{*}\right)=\bigoplus_{p, q} \Omega^{q}\left(G^{p}\right)
$$

of $q$-forms on the $p$-fold products. $G$ being a group, so is $G^{p}$ and hence we can identify

$$
\Omega^{q}\left(G^{p}\right)=C^{\infty}\left(G^{p} ; \Lambda^{q}\left(\bar{g}^{p}\right)\right)
$$

where $\Lambda^{q}$ denotes the $q$ th exterior power and $\bar{g}^{p}$ the dual of the Lie algebra of $G^{p}$. The usual approximation of smooth by continuous functions then gives the homology for fixed $q$ as $H_{c}\left(G ; H\left(\Lambda^{q}\left(\bar{g}^{p}\right)\right)\right)$. Standard homological algebra gives the homology coefficients as $S^{q}(\bar{g})$. The edge map $H(B G) \rightarrow$ $H_{c}(G)$ is the map induced by $u \rightarrow u \circ \Delta$ above.

For a reductive Lie group, we can further identify $E_{1}$ as

$$
H_{c}(G) \otimes S^{*}(\bar{g})^{G}
$$

the latter factor denoted classically as $I_{G}$, the invariant polynomials.

Working entirely from the Lie algebra point of view, Kamber and Tondeur [32] had earlier produced a formally isomorphic spectral sequence with $E_{1} \approx H^{*}(g, K) \otimes S^{*}(\bar{g})^{G}$. Using their techniques, I have been able to show 
the two spectral sequences are naturally isomorphic with the $E_{1}$ isomorphism being the van Est result. Their techniques also permit the natural identification of these spectral sequences with the Leray-Serre spectral sequence of the fibration

$$
G_{u} / K \rightarrow B K \rightarrow B G_{u} \text {. }
$$

The advantage of Bott's approach is that it works for a not necessarily reductive Lie group.

Consider for example $G=G L(n, R)$. We have already stated $H_{c}(G)$, and $I_{G}$ is known to be $R\left[c_{1}, \ldots, c_{n}\right]$ where $c_{i}$ is of dimension $2 i$ [23]. In this case we also know $H(B G) \approx R\left[c_{2}, c_{4}, \ldots, c_{2[n / 2]}\right]$ and hence conclude, up to a change of basis, $d_{i} h_{i}=c_{i}$ for $i$ odd. Portraying this in terms of generators only, we have:

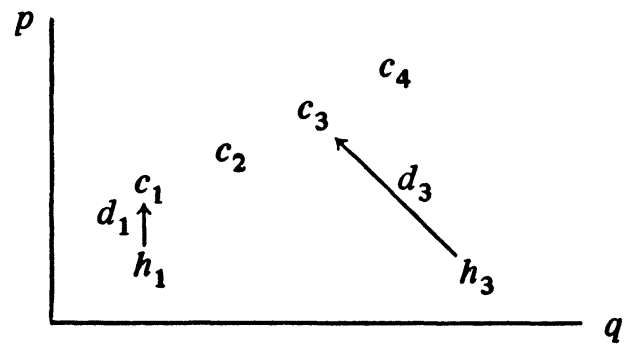

7. Continuous cohomology of topological groupoids. Now all of this would be history, mostly ancient, were it not for new, powerful and applicable generalizations. Lie was not interested in groups alone. Because of the relevance to partial differential equations, Lie was interested in smooth transformations of one parameterized space to another. In modern terminology, a suitable setting is that of local diffeomorphisms of a manifold; it is sufficient to consider $R^{n}$. Since two such transformations cannot always be composed (we need the range of one to be within the domain of the next) we have not a group but a pseudogroup. From the point of view of differential equations, it is the infinitesimal behaviour that is paramount, so we pass to equivalence classes where two transformations on neighborhoods of $x$ are equivalent if they agree on some smaller neighborhood. The equivalence classes are called germs. The collection of equivalence classes now form a groupoid (each germ has an inverse) called $\Gamma_{n}$. It inherits a topology from the original manifold, $R^{n}$; indeed $\Gamma_{n}$ is locally diffeomorphic to $R^{n}$ though not paracompact or even Hausdorff. When defined, composition is still associative and continuous, and since local diffeomorphisms have continuously varying local inverses, $\Gamma_{n}$ is a topological groupoid.

[In what follows, we limit ourselves to groupoids because of their intrinsic interest, although the inverses play no significant role and the formal definitions go through for topological pseudogroups.]

For topological groupoids $\Gamma$, the above definitions of $H_{c}$ and $B$ make sense if we make just one modification. Instead of $G^{p}$, we use $\Gamma^{(p)} \subset \Gamma^{p}$ where $\left(\gamma_{1}, \ldots, \gamma_{p}\right) \in \Gamma^{(p)}$ iff $\gamma_{1} \circ \ldots \circ \gamma_{p}$ is defined. Now $H_{c}^{*}(\Gamma ; A)$ is defined as the cohomology of the cochain group 


$$
C_{c}^{p}(\Gamma ; A)=\left\{\text { continuous } f: \Gamma^{(p)} \rightarrow A\right\}
$$

with respect to the coboundary we've been using. To generalize to coefficients in a continuous $\Gamma$ module $A$, we define the latter to mean the following: Let $\Gamma^{(0)}$ denote the space of right identities or sources of $\Gamma$ and require $A$ to come equipped with an onto map $\pi: A \rightarrow \Gamma^{(0)}$ and a map $A \times_{\Gamma^{(0)}} \Gamma \rightarrow A$ satisfying the associativity condition. For example, let $\Gamma=$ \{germs of local diffeomorphisms of a manifold $M$ \} and $A=T^{*} M$, the cotangent bundle, with $\pi$ : $A \rightarrow \Gamma^{(0)}=M$ the standard projection. Now restrict

$$
C_{c}^{p}(\Gamma ; A)=\left\{\text { continuous } f: \Gamma^{(p)} \rightarrow A \mid \pi f=\mathrm{id}_{\gamma_{p}}\right\}
$$

so that the last term of $\delta$ makes sense: $f\left(\gamma_{1}, \ldots, \gamma_{p}\right) \cdot \gamma_{p+1}$ is defined.

Similarly $B \Gamma$ is defined as a quotient of $\Delta^{p} \times \Gamma^{(p)}$ by the same relation as for $B G$. Now $B \Gamma_{n}$ turns out to be relevant to the classification of foliations of manifolds. A foliation can be defined as a decomposition of a manifold $M$ into manifolds $L_{\alpha}$ of codimension $n$ such that locally the decomposition is equivalent to that of $R^{m}$ by coordinate planes of codimension $n$. A crucial example is provided by solution sets of differential equations.

The cohomology $H\left(B \Gamma_{n}\right)$ then gives rise to characteristic classes for such foliations.

Here we must distinguish between the de Rham cohomology $H$ and the singular cohomology $\mathcal{H}$. Although neither Hausdorff nor paracompact, $\Gamma_{n}^{(p)}$ is locally diffeomorphic to $R^{n}$ and hence has a tangent bundle and $\Omega^{*}\left(\Gamma_{n}^{(p)}\right)$ is defined. Since the de Rham theorem is unknown for $\Gamma_{n}$ we can make no comparison with $\mathcal{H}\left(B \Gamma_{n}\right)$ but the cohomology of $\Omega^{*}\left(\Gamma^{(*)}\right)$ is isomorphic to $H\left(B \Gamma_{n}\right)$. Although the map into $\mathcal{H}\left(B \Gamma_{n}\right)$ is not known to be an isomorphism, it does give characteristic classes. On the other hand for any manifold $M$ mapped into $B \Gamma_{n}$, the de Rham theorem for $M$ guarantees that the kernel of $H\left(\Omega^{*}\left(\Gamma^{(*)}\right)\right) \rightarrow \mathcal{H}\left(B \Gamma_{n}\right)$ is mapped to zero in $H(M) \approx \mathcal{H}(M)$.

Bott's analysis of $\Omega^{*}\left(G^{*}\right)$ carries over to $\Omega^{*}\left(\Gamma^{(*)}\right)$ with one important modification, as Shulman and I worked out [55]. Notice that since the tangent bundle to $\Gamma_{n}^{(p)}$ is induced from that of $R^{n}$, it trivializes and

$$
\Omega^{q}\left(\Gamma_{n}^{(p)}\right)=C^{\infty}\left(\Gamma_{n}^{(p)} ; \Lambda^{q}\left(R^{n}\right)\right)
$$

Since the exterior powers of $R^{n}$ alone and not iterated products occur, the symmetric algebra does not appear. Indeed

$$
E_{1}^{p, q} \approx H_{c}^{p}\left(\Gamma ; \Lambda^{q}\left(R^{n}\right)\right)
$$

Since this talk was given, this group has been computed in part; we return to it shortly.

Now there is a very nice map $\Gamma_{n} \rightarrow G L(n, R)=G$ given by taking the differential at the source. This respects composition; we have induced maps $B \Gamma_{n} \rightarrow B G$ and $\Omega^{*}\left(G^{*}\right) \rightarrow \Omega^{*}\left(\Gamma^{(*)}\right)$. The vanishing of $\Omega^{q}\left(\Gamma_{n}^{(*)}\right)$ for $q>n$ together with the spectral sequence information above gives the Bott vanishing theorem: $R\left[c_{2}, \ldots, c_{2[n / 2]}\right] \rightarrow H^{*}\left(B \Gamma_{n}\right)$ is zero in degrees greater than $2 n$.

It is rather tempting to consider the factorization

$$
\Omega^{*}\left(G^{*}\right) \rightarrow \Omega^{*}\left(G^{*}\right) f / \text { forms of } \operatorname{deg}>n \rightarrow \Omega^{*}\left(\Gamma^{(*)}\right)
$$

and to compute the cohomology of the middle term, call it $B$. Look at 


$$
E_{1}^{p, q}(B)= \begin{cases}E_{1}^{p, q}(G), & q \leqslant n, \\ 0, & q>n .\end{cases}
$$

The differentials are otherwise the same as in $E_{1}\left(\Omega^{*} G^{*}\right)$ and the results can be compared. A worthwhile summary is given by considering

$$
W O_{n}=\Lambda\left(h_{1}, h_{3}, \ldots, h_{\langle n\rangle}\right) \otimes R\left[c_{1}, \ldots, c_{n}\right] / \text { total deg }>2 n \text { with } d h_{i}=c_{i}, i \text { odd, }
$$

then we can show $H\left(W O_{n}\right) \approx H(B)$, so we have

$$
H(B G L(n, R)) \rightarrow H\left(W O_{n}\right) \rightarrow H\left(B \Gamma_{n}\right) .
$$

Until recently, the cohomology of $B \Gamma_{n}$ was studied by constructing specific foliations with nontrivial characteristic classes. The first detected and most famous is $h_{1} c_{1}^{n}$, called the Godbillon-Vey class [22], [58]. Its direct description in terms of de Rham forms enables it to be computed and shown to be nontrivial for a foliation on the unit tangent sphere bundle to a compact $M^{n}$ of constant negative curvature. To identify other classes, Vey [21] has given a basis for $H\left(W O_{n}\right)$ (all products are zero) consisting of all the classes $h_{i_{1}} h_{i_{2}} \cdots h_{i_{r}} \otimes c_{j_{1}} \cdots c_{j_{s}}$ such that $i_{1}<i_{2}<\cdots$ and $j_{1} \leqslant j_{2} \leqslant \cdots$ and further $i_{1}+\sum j_{k}>n$ while $i_{1} \leqslant j_{1}$. Denote such a class as $h_{I} c_{J}$ with $I=$ $\left(i_{1}, \ldots\right), J=\left(j_{1}, \ldots\right)$. By more homotopy theoretic methods, Thurston [58] has shown $c_{2}$ is nonzero in $H^{4}\left(B \Gamma_{n}\right), n>1$. By subtle improvements of standard techniques, Morita [46] then showed the independence of classes $h_{1} c_{1}^{r} c_{2}^{s}$ for $r+2 s=n, r>0$ in $H^{2 n+1}\left(B \Gamma_{n}\right)$. Meanwhile Kamber and Tondeur [33] and more recently Baker [1] were able to show large numbers of the $h_{I} c_{J}$ were linearly independent. The essential geometric technique is the construction of foliations induced from right $H$-coset foliations on a Lie group $G$ by passing to the quotient $\Gamma \backslash G$ for $\Gamma$ a suitable discrete subgroup acting on the left. Indeed Roussarie's example for the Godbillon-Vey class is of this form.

Finally John Petro has just shown the map $H\left(W O_{n}\right) \rightarrow \mathcal{H C}\left(B \Gamma_{n}\right)$ is a monomorphism. His proof uses an analogous spectral sequence for $\Gamma_{0,0}$, the germs of local diffeomorphisms with source and target at the origin (topologized as a quotient of $\left.\Gamma_{n}\right)$. A comparison of $H_{c}\left(\Gamma_{0,0}\right)$ with $H_{c}\left(J_{n}\right)$ where $J_{n}$ is the space of infinite jets (at the origin) of local $C^{r}$-diffeomorphisms of $R^{n}$ yields a monomorphism $H^{*}\left(W O_{n}\right) \rightarrow \mathcal{H}^{*}\left(B \Gamma_{0,0}\right)$ which in turn is isomorphic to $\mathcal{H}^{*}\left(B \Gamma_{n}\right)$.

Meanwhile Fuks [69] has announced the injectivity of $H\left(W O_{n}\right) \rightarrow \mathcal{H}\left(B \Gamma_{n}\right)$ and related maps, using rather different methods.

There is one further aspect of these classes where our knowledge is incomplete. Some of these classes, that of Godbillon-Vey for example, can and do vary continuously. That is, there is a continuous family of foliations $F_{t}$ on a manifold $M$ of dimension $2 n+1$ such that the corresponding Godbillon-Vey classes $h_{1} c_{1}^{n}$ for $F_{t}$, when evaluated on the fundamental cycle of $M$, run over all real values. Of course the family does not form an integrable homotopy for then the value would have to be constant. In terms of maps, we have $M_{\overrightarrow{\phi_{1}}} B \Gamma_{n}$ lifting to a map $M \times I \rightarrow B \Gamma_{n+1}$, but not to $M \times I \rightarrow B \Gamma_{n}$. The classes in $B \Gamma_{n}$ which pull back from $B \Gamma_{n+1}$ cannot vary and are therefore called rigid; the ones which can vary continuously are not in the 
image. In terms of the Vey basis, the latter are precisely those $h_{I} c_{J}$ with $i_{1}+\Sigma j_{k}=n+1$. In the Bott spectral sequence, they occur in $E_{1}^{p, q}$ with nontrivial differential $d_{r}$ such that $q+r=n+1$. Thus they are cycles if we truncate above $n$, but not if we truncate above $n+1$.

All of these special $h_{I} c_{J}$ then are potentially capable of varying continuously, but we have only limited knowledge of which do and even less as to how independently the various classes can vary. The most extensive result I am aware of is due to Heitsch [27]. He shows the classes in $H^{4 k-1}\left(W O_{2 k-1}\right)$ can vary over a range of values at least as big as $R^{k}$. [See also [51].]

ADDED IN PROOF. Fuks [69] has announced that in the case of foliations with trivial normal bundle all the potentially variable $h_{I} c_{J}$ do vary.

8. Continuous cohomology of vector fields. In addition to germs of local diffeomorphisms, the group Diff $M$ of all diffeomorphisms of a manifold $M$ is of great interest. Motivated by van Est's result, one might look for a suitable Lie algebra for Diff $M$. Gel'fand and Fuks in a series of remarkable papers [19], [20] turned their attention to the Lie algebra $\mathscr{X}(M)$ of vector fields on $M$. Excellent surveys of their work, particularly in relation to foliations, are provided by Bernšteĭn and Rosenfel'd [3] and by Bott [50]. We will comment on their work only in relation to the preceding sections here.

Since $\mathcal{X}(M)$ is itself infinite dimensional, continuity is not necessarily a consequence of linearity, so Gel'fand and Fuks consider the complex

$$
C_{c}^{q}(\mathcal{X}(M) ; A)=\operatorname{Alt}_{c}^{q}(\mathcal{X}(M) ; A)
$$

of alternating multilinear maps continuous with respect to the topology. The coboundary is as in $\$ 5$. Denote the cohomology by $H_{c}$. They also consider the space of formal vector fields $A_{n}$ on $R^{n}$ : vector fields of the form $\Sigma p_{i}\left(x_{1}, \ldots, x_{n}\right) \partial / \partial x_{i}$ where $p_{i}$ is a formal power series. An alternate notation is

$$
\mathbb{Q}_{n}=R\left[\left[x_{1}, \ldots, x_{n}\right]\right] \otimes\left\langle\partial / \partial x_{1}, \ldots, \partial / \partial x_{n}\right\rangle
$$

where \langle\rangle denotes the vector space spanned. There is an explicit and canonical map

$$
W O_{n} \rightarrow C_{c}^{*}\left(\mathbb{Q}_{n} ; R\right)
$$

which they show induces an isomorphism in cohomology. Thus we have a factorization $H(B G) \rightarrow H_{c}\left(A_{n}\right) \rightarrow H\left(B \Gamma_{n}\right)$, which can be interpreted directly on the cochain level in terms of the associated infinite jet bundle [18].

ADDED IN PROOF. It is this map $H_{c}\left(A_{n}\right) \rightarrow H\left(B \Gamma_{n}\right)$ as well as many others of a similar form: $H_{c}(\mathrm{~g}) \rightarrow H(B g)$ for which Fuks [69] has outlined a proof of injectivity.

9. Coda. We began with the cohomology of abstract groups. Given a topological group $G$, we can ignore the topology and consider the corresponding discrete group $G^{\delta}$. For $G=G L(n, R)$ we have

$$
B G^{\delta} \rightarrow B \Gamma_{n} \rightarrow B G \text {. }
$$

Notice that the continuous cohomology $H_{c}\left(G^{\delta}\right)$ is the abstract group 
cohomology $\approx H\left(B G^{\delta}\right)$ so we have

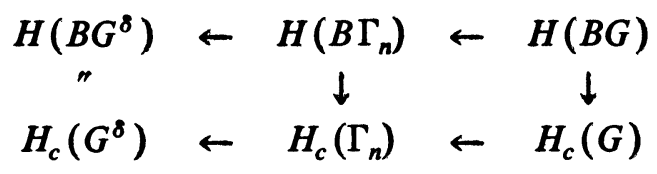

where the vertical maps can be interpreted as the edge morphisms of the Bott spectral sequence. The composite $H_{c}(G) \rightarrow H_{c}\left(G^{\delta}\right)$ is known to be a monomorphism (by results of Borel and Selberg showing the existence of enough discrete subgroups of $G$ to detect all the continuous cohomology [4]). In general $H_{c}(G)$ is intermediate between $H(B G)$ and $H\left(B G^{\delta}\right)$.

Finally we can think of $B G^{\delta}, B G$ as being the same space with two topologies (see \$6). Bott and Haefliger consider a new cohomology using both topologies and denoted $\mathcal{H}_{c}\left(B G^{\delta} \rightarrow B G\right)$. It is defined in terms of the singular simplices of $B G^{\delta}$ by considering cochains which are continuous with respect to the $B G$-induced topology on the space of singular simplices in $B G^{\delta}$ [49], cf. [67]. (With just one topology and reasonably nice spaces [30], this reduces to ordinary singular cohomology.) They prove

$$
H_{c}(G) \approx \mathcal{H}_{c}\left(B G^{\delta} \rightarrow B G\right) .
$$

M. Mostow has since thoroughly studied this notion, both axiomatically and through illustrative examples, and clarified its relation to foliations [49].

Thus continuous group theoretic cohomology $H_{c}(G)$ is intimately and intricately related in various ways to the cohomology of the classifying space $B G$.

It is interesting to compare $H^{*}(B G ; Z)$ and $H^{*}(B G ; R)$ via Borel and continuous cohomology.

$$
\begin{aligned}
& H^{*-1}(B G ; R / Z) \rightarrow H^{*}(B G ; Z) \rightarrow H^{*}(B G ; R) \\
& \downarrow \approx \quad H_{c}(G ; R) \\
& H_{\text {Borel }}^{*-1}(G ; R / Z) \rightarrow H_{\text {Borel }}(G ; Z) \rightarrow H_{\text {Borel }}(G ; R)
\end{aligned}
$$

For a compact Lie group $G, H_{c}(G ; R)=0$ so the machinery of functional cohomology operations can be applied to (the real image of) any integral cohomology class $u$, for example Chern classes in $H^{*}(B U(n) ; R)$. The result is the Simons-Cheegar character of $u$ [14]. Explicit formulas in terms of integration of forms are given also by Baker [2]. For a compact Lie group $G$, the differential characters correspond precisely to $H_{\text {Borel }}(G ; R / Z)$.

\section{REFERENCES}

1. D. Baker, On a class of foliations and the evaluations of their characteristic classes, Thesis, SUNY, Stony Brook, 1976.

2. Differential characters and Borel cohomology, Topology 16 (1977), 441-449.

3. I. N. Bernstein and B. I. Rosenfel'd, Homogeneous spaces of infinite-dimensional Lie algebras and characteristic classes of foliations, Russian Math Surveys 28 (1973), 107-142. 
4. A. Borel, Compact Clifford-Klein forms of symmetric spaces, Topology 2 (1963), 111-122.

5. , Seminar notes IAS 1976-1977.

6. R. Bott (with L. Conlon), Lectures on characteristic classes, Lecture Notes in Math., vol. 279, Springer-Verlag, Berlin and New York, 1972, pp. 1-94.

7. R. Bott, On the Chern-Weil homomorphism and the continuous cohomology of Lie groups, Advances in Math. 11 (1973), 289-303.

8. , Some remarks on continuous cohomology, Proc. Internat. Congress on Manifolds (Tokyo, 1973), Univ. of Tokyo Press, Tokyo, 1975, pp. 161-170.

9. R. Bott, H. Shulman and J. Stasheff, On the de Rham theory of classifying spaces, Advances in Math. 20 (1976), 43-56.

10. L. G. Brown, Extensions of topological groups, Pacific J. Math. 39 (1971), 71-78.

11. H. Cartan, Notions d'algèbre différentielle, Colloq. de Topologie Bruxelles, 1950, pp. 15-27.

12. J-L. Cathelineau, Un objet simplicial associé à un recouvrement d'un groupe topologique, Université de Poitiers (preprint).

13. W. Casselman and D. Wigner, Continuous cohomology and a conjecture of Serre's, Invent. Math. 25 (1974), 199-211.

14. J. Cheeger and J. Simons, Jr., Differential characters and geometric invariants, SUNY, Stony Brook, (preprint). SUNY, Stony Brook, cf. C. Roger, Caractères différentiels, Lecture Notes in Math., vol. 484, Springer-Verlag, Berlin and New York, 1975, pp. 162-178.

15. C. Chevalley and S. Eilenberg, Cohomology theory of Lie groups and Lie algebras, Trans. Amer. Math. Soc. 63 (1948), 85-124.

16. A. Dold and R. Lashof, Principal quasifibrations and fibre homotopy equivalence of bundles, Illinois J. Math. 3 (1959), 285-305.

17. J. L. Dupont, Simplicial de Rham cohomology and characteristic classes of flat bundles, Topology 15 (1976), 233-246.

18. D. B. Fuks, Characteristic classes of foliations, Russian Math. Surveys 28 (1973), 1-16.

19. I. M. Gel'fand and D. B. Fuks, Cohomologies of the Lie algebra of tangent vector fields of $a$ smooth manifold, Functional Anal. Appl. 3 (1969), 194-210.

20. 322-337.

21. C. Godbillon, Cohomologie d'algèbres de Lie de champs de vecteurs formels, Séminaire Bourbaki 421 (1972/73).

22. C. Godbillon and J. Vey, Un invariant des feuilletages de codimension un, C. R. Acad. Sci. Paris 273 (1971), 92.

23. W. Greub, S. Halperin and R. Van Stone, Connections, curvature and cohomology. III, Academic Press, New York, 1976, p. 254.

24. A. Haefliger, Sur les classes caracteristiques des feuilletages, Séminaire Bourbaki 412 (1972/73).

25. __ Feuilletages sur les variétés ouvertes, Topology 9 (1970), 183-194.

26. J. Heitsch, Deformations of secondary characteristic classes, Topology 12 (1973), 381-388.

27. , Independent variation of secondary classes (manuscript).

28. A. Heller, Principal bundles and group extensions with applications to Hopf algebras, J. Pure Appl. Algebra 3 (1973), 219-250.

29. G. P. Hochschild and G. D. Mostow, Cohomology of Lie groups, Illinois J. Math. 6 (1962), $367-401$.

30. S-T Hu, Cohomology rings of compact connected groups and their homogeneous spaces, Ann. of Math (2) 55 (1952), 391-419.

31. Cohomology theory in topological groups, Michigan Math. J. 1 (1952), 11-59.

32. F. Kamber and $\mathrm{Ph}$. Tondeur, Characteristic invariants of foliated bundles, Manuscripta Math. 11 (1974), 51-89.

33. _ On the linear independence of certain cohomology classes of $B \Gamma$ (preprint).

34. M. Lazard, Groupes analytiques p-adiques, Publ. Math. IHES 26 (1975).

35. S. Lie, Om en classe geometriske Transformationen, Ges. der Wiss. zu Christiania, 1870.

36. , Theorie der Transformations-gruppen, Teubner, Leipzig, 1890.

37. G. Mackey, Les ensembles Boreliens et les extension des groupes, J. Math. Pures Appl. 36 (1957), 171-178. 
38. S. Mac Lane, Homology, Die Grundlehren der Math. Wissenschaften, vol. 114, SpringerVerlag, Berlin and New York, 1963.

39. _ Retiring Presidential Address, Bull. Amer. Math. Soc. 82 (1976), 1-40.

40. ㄴ The work of Samuel Eilenberg in Topology, Algebra, topology, and category theory: a collection of papers in honor of Samuel Eilenberg, Academic Press, New York, 1976, p. 137.

41. R. J. Milgram, The bar construction and abelian H-spaces, Illinois J. Math 11 (1967), 242-250.

42. J. W. Milnor, Construction of universal bundles. II, Ann. of Math. (2) 63 (1956), 430-436.

43. C. C. Moore, Extensions and low dimensional cohomology theory of locally compact groups. I, II, Trans. Amer. Math. Soc. 113 (1964), 40-86; III, IV, Trans. Amer. Math. Soc. 221 (1976), 1-58.

44. Group extensions and group cohomology, Battelle Seattle Summer Rencontre,

Lecture Notes in Math., Springer-Verlag, Berlin and New York, 1970, pp. 17-36.

45. J. C. Moore, Algèbre homologique et homologie des espaces classifiants, Séminaire H. Cartan (1959/60).

46. S. Morita, A remark on the continuous variation of secondary characteristic classes for foliations (preprint).

47. P. S. Mostert, Local cross section in locally compact groups, Proc. Amer. Math. Soc. 4 (1953), 645-649.

48. G. D. Mostow, Cohomology of topological groups and solvmanifolds, Ann. of Math. (2) 73 (1961), $20-48$.

49. M. A. Mostow, Continuous cohomology of spaces with two topologies, Mem. Amer. Math. Soc. No. 175 (1976).

50. M. Mostow and J. Perchik, Notes on Gel'fand-Fuks cohomology and characteristic classes (Lectures by Raoul Bott), New Mexico State Univ., 1973.

51. O. H. Rasmussen, Non-vanishing and continuous variation of exotic characteristic classes for foliations, Thesis, Princeton Univ., 1974.

52. G. Segal, Categories and cohomology theories, Topology 13 (1974), 293-312.

53. __ Cohomology of topological groups, Symposia Mathematica IV (1970), 377-387.

54. J-P. Serre, Cohomologie Galoisienne, Lecture Notes in Math., vol. 5, Springer-Verlag, Berlin and New York, 1973.

55. H. Shulman and J. D. Stasheff, de Rahm theory for Br, differential topology, foliations and Gelfand-Fuks cohomology, Proc. Topology Sympos. PUC, Rio de Janeiro, 1976, Lecture Notes in Math., vol. 652, Springer-Verlag, Berlin and New York, 1978.

56. J. D. Stasheff, $H$-spaces from a homotopy point of view, Lecture Notes in Math., vol. 161, Springer-Verlag, Berlin and New York, 1970 (Chapter 6. The bar construction spectral sequence.) 57. __ On extensions of H-spaces, Trans. Amer. Math. Soc. 105 (1962), 126-135.

58. W. Thurston, Foliations and groups of diffeomorphism, Bull. Amer. Math. Soc. 80 (1974), 304-307.

59. The theory of foliations of codimension greater than one, Differential Geometry

(Proc. Sympos. Pure Math., Stanford, 1973), Amer. Math. Soc., Providence, R. I., 1975.

60. Variations of the Godbillon-Vey invariant in higher dimensions (to appear).

61. W. T. van Est, A generalization of the Cartan-Leray spectral sequence. I, II, Proc. Kon. Neder. Akad. Wetensch. A 61 (1958), 399-413.

62. , Group cohomology and Lie algebra cohomology in Lie groups. I. II. Proc. Kon. Neder. Akad. Wetensch. A 56 = Indag. Math. 15 (1953), 484-504.

63. , On the algebraic cohomology concepts in Lie groups. I, II, Proc. Kon. Neder. Akad. Wetensch. A 58 (1955), 225-233, 286-294.

64. Une application d'une methode de Cartan-Leray, Proc. Kon. Neder. Akad. Wetensch. A 58 = Indag. Math. 17 (1955), 542-544.

65. C. Watkiss, Thesis, Univ. of Toronto, 1975.

66. D. Wigner, Algebraic cohomology of topological groups, Trans. Amer. Math. Soc. 178 (1973), 83-93.

67. A. Haefliger, Differentiable cohomology, Cours donné au CIME (1970).

68. H. Samelson, Zum wissenschaftlichen Werk von Heinz Hopf, Jber. Deutsch. Math.-Verein. 78 (1976), 126-146. 
69. D. Fuchs, Non-trivialité des classes caractéristiques de g-structures. Applications aux classes caractéristiques de feuilletages, C. R. Acad. Sci. Paris 284 (1977), 1017-1019.

, Applications aux variations des classes caractéristiques de feuilletages, C. R. Acad. Sci.

Paris 284 (1977), 1105-1107.

Department of Mathematics, Temple University, Philadelphia, Pennsylvania 19122

Current address: Department of Mathematics, University of North Carolina, Chapel Hill, North Carolina 27514 